\title{
Fatores de risco associados às quedas em idosos e reflexões acerca de sua prevenção: um estudo de revisão
}

\author{
Risk factors associated with falls in elderly and reflexions on prevention: \\ a review study \\ Factores de riesgo asociados a las caídas en ancianos y reflexiones sobre la \\ prevención: un estudio de revisión \\ Paulo Vinicio Neves de ALENCAR ${ }^{1}$ \\ Yago Tavares PINHEIRO ${ }^{1}$ \\ Alysson Aranha dos SANTOS ${ }^{2}$ \\ Maria Gabrielly Fernandes de Souza NONATO ${ }^{1}$ \\ Dayvianne Cecília Ribeiro Teixeira MOREIRA ${ }^{1}$ \\ Giane Dantas de Macedo FREITAS ${ }^{3}$
}

${ }^{1}$ Fisioterapeutas pela Faculdade Maurício de Nassau, UNINASSAU, 58030-000, João Pessoa - PB, Brasil

${ }^{2}$ Médico pela Universidade Federal da Paraíba, UFPB, 58051-900, João Pessoa - PB, Brasil

${ }^{3}$ Professora Doutora do Curso de Fisioterapia da Faculdade Maurício de Nassau, UNINASSAU, 58030-000, João Pessoa - PB, Brasil

\begin{abstract}
Resumo
Introdução: o envelhecimento é um processo natural, progressivo e irreversível, entretanto algumas mudanças interferem na qualidade de vida do idoso, tornando- o mais suscetível aos processos patológicos. As quedas em idosos são eventos frequentes, sendo considerado um problema de saúde pública. Objetivo: analisar os principais fatores de risco para quedas em idosos dispostos na literatura científica e apresentar uma reflexão sobre sua prevenção. Métodos: Trata-se de uma revisão de literatura a partir de estudos publicados em periódicos indexados nas bases de dados SciELO, LILACS, MEDLINE e BVS, em português, no período de 2010 a 2015 e que atendessem à questão norteadora. Assim, foram analisados 09 artigos que rigorosamente atenderam aos critérios de elegibilidade estabelecidos. Resultados: observou-se que as quedas são comuns no cotidiano dos idosos, as quais foram fortemente associadas ao uso de medicamentos, tontura, desequilíbrio, falta de força muscular e inadequações no ambiente físico e social, e sua prevenção se torna essencialmente importante, uma vez que esses eventos se caracterizam como problemas de saúde pública. Conclusão: este estudo possibilitou o intercâmbio entre os conhecimentos teóricos em gerontologia e a percepção no tocante à prevenção de risco de quedas em idosos, fazendo-se necessário e abrangente, visto que é essa população se encontra em ascensão significativa nos últimos anos.

Descritores: Idoso; Equilíbrio Postural; Acidentes por Quedas; Prevenção \& Controle; Saúde Pública.
\end{abstract}

\begin{abstract}
Introduction: Aging is a natural, progressive and irreversible process, though some changes affect the quality of life of the elderly, making it more susceptible to disease processes. Falls in the elderly are frequent events, and is considered a public health problem. Objective: To analyze the major risk factors for falls in the elderly arranged in the scientific literature and present a reflection on prevention. Methods: This is a literature review from studies published in journals indexed in databases SciELO, LILACS, MEDLINE and BVS in Portuguese, in the period from 2010 to 2015 and that met the guiding question. Thus, we analyzed 09 articles that strictly met the established eligibility criteria. Results: It was observed that the falls are common in the daily lives of the elderly, which were strongly associated with the use of drugs, dizziness, unsteadiness, lack of muscle strength and inadequacies in the physical and social environment, and prevention becomes essentially important, since these events are characterized as public health problems. Conclusion: This study enabled the exchange between theoretical knowledge in gerontology and awareness regarding the prevention of risk of falls in the elderly, making it necessary and comprehensive, as it is this population is a significant rise in recent years.

Descriptors: Aged; Postural Balance; Acidental Falls; Prevention \& Control; Public Health.
\end{abstract}

\section{Resumen}

Introducción: El envejecimiento es un proceso natural, progresivo e irreversible, aunque algunos cambios afectan a la calidad de vida de las personas mayores, por lo que es más susceptible a los procesos de enfermedad. Caídas en los ancianos son frecuentes los acontecimientos, y se considera un problema de salud pública. Objetivo: Analizar los principales factores de riesgo de caídas en los ancianos dispuestos en la literatura científica y presenta una reflexión sobre la prevención. Métodos: Se trata de una revisión de la literatura de los estudios publicados en revistas indexadas en bases de datos SciELO, LILACS, MEDLINE y BVS en portugués, en el período de 2010 a 2015 y que se reunió la cuestión de guía. Por lo tanto, se analizaron 09 artículos que cumplían estrictamente los criterios de elegibilidad establecidos. Resultados: Se observó que las caídas son comunes en la vida diaria de las personas de edad, los cuales fueron fuertemente asociados con el uso de drogas, mareos, inestabilidad, falta de fuerza muscular y las deficiencias en el entorno físico y social, y la prevención se convierte esencialmente importante, ya que estos eventos se caracterizan por problemas de salud pública. Conclusión: Este estudio permitió el intercambio entre los conocimientos teóricos en gerontología y la sensibilización con respecto a la prevención del riesgo de caídas en los ancianos, por lo que es necesaria e integral, ya que es esta población es un aumento significativo en los últimos años.

Descriptores: Anciano; Balance Postural; Accidentes por Caídas; Prevención \& Control; Salud Pública.

\section{INTRODUÇÃO}

No Brasil, a população idosa vem crescendo significativamente e com isso surge a necessidade de discussão a respeito de cuidados voltados a essa população, uma vez que o envelhecimento é um processo natural, progressivo e irreversível que interfere diretamente nas condições biológicas e funcionais ${ }^{1}$.

Assim, as quedas em idosos surgem como eventos frequentes e comuns nessa etapa da vida, sendo responsáveis por dois terços das mortes acidentais, principalmente em indivíduos com mais de 65 anos de idade, representando grande causa de morbidade e mortalidade nessa faixa etária. Atualmente, considera-se um problema de saúde pública devido à sua alta incidência e às consequências negativas em relação à qualidade de vida ${ }^{2}$.

As consequências das quedas para os idosos são inúmeras, desde alterações biológicas até fraturas 
importantes, interferindo no psicossocial do individuo, nas atividades diárias, entre outros. Comumente, tem-se dado uma atenção maior às lesões físicas causas pelas quedas, já que estas se apresentam perigosas, inclusive podendo levar ao óbito quando não tratadas. Esses prejuízos vêm atrelados também a internações e tratamentos que despendem alto custo financeiro para as famílias ${ }^{3}$.

Conforme a Politica Nacional da Saúde do Idoso, comparando o envelhecimento benéfico com a conservação do individuo idoso no seu recinto familiar e com sua capacidade funcional estável, uma elaboração de um programa que estimule a conscientização da sociedade e dos indivíduos idosos que trabalhem medidas para diminuir risco de quedas é urgente ${ }^{4}$.

Nesse contexto, a prevenção faz-se necessária no sentido de minimizar sequelas físicas, sociais e psicológicas decorrente de quedas, fomentando a adequação de serviços de saúde, bem como dos profissionais envolvidos no atendimento multidisciplinar dessas pessoas. Por conseguinte, percebe-se a necessidade de estudos direcionados a essa temática com intuito de reduzir a incidência de quedas e suas consequências e de oferecer ao público conhecimentos básicos de orientação sobre fatores de risco de quedas em ambientes domiciliares ${ }^{5}$.

Portanto, o presente estudo objetivou analisar os fatores de risco para ocorrência de quedas, analisar as consequências funcionais e psicológicas nessa população, bem como realizar um levantamento acerca da prevenção desses eventos.

\section{MATERIAL E MÉTODO}

Para atingir o objetivo proposto, optou-se pela realização de um estudo exploratório do tipo revisão de literatura. A questão norteadora para o desenvolvimento foi: quais os fatores de risco, as consequências e as formas preventivas associadas às quedas em idosos?

A coleta dos estudos se deu no período de fevereiro a abril de 2016 nas bases de dados on line SciELO, LILACS, MEDLINE e BVS por meio do cruzamento dos descritores "idoso", "equilíbrio", "quedas" e seus respectivos sinônimos em inglês.

Em termos de elegibilidade foram estabelecidos como critérios de inclusão estudos publicados nas referidas bases de dados, em português, no período de 2010 a 2015 e que atendessem à questão norteadora.

O material inicialmente catalogado para a pesquisa foi submetido a uma análise criteriosa levando-se em consideração a pertinência das temáticas estudadas com o presente estudo onde foram incluídas referências que abordam elementos relacionados aos fatores de risco para a ocorrência de quedas, suas complicações e principalmente como atuar na sua prevenção.

A partir da metodologia descrita, um total de 09 (nove) estudos foi selecionado para compor a amostra da presente revisão de literatura. Para síntese e análise desses estudos, foi confeccionado um guia de integração contendo informações sobre autor/ano, título, periódico, objetivo e resultados. Por fim, a apresentação e discussão dos resultados foram realizadas de forma descritiva.

\section{RESULTADOS}

A presente revisão de literatura analisou 09 (nove) artigos que rigorosamente atenderam aos critérios de elegibilidade estabelecidos. O quadro 1 apresenta o guia de integração mencionado anteriormente com a síntese dos estudos incluídos.
Assim, 09 publicações foram de relevância para esta revisão, uma vez que atenderam aos critérios de inclusão pré-estabelecidos e trouxeram contribuições importantes ao desenvolvimento do estudo.

A partir dos resultados, observou-se que as quedas são corriqueiras no dia a dia dos idosos, não sendo um fator isolado do envelhecimento humano. Os principais fatores associados às quedas descritos nos artigos foram a presença das escadas, problemas de locomoção (sequelas de doenças), diminuição do auto cuidado, o papel familiar e, sobretudo, diminuição da qualidade de vida, ou seja, isso demonstra que os fatores intrínsecos e extrínsecos que são relacionados as quedas na população idosa se encontram inseridos em todos os estudos selecionados, sendo a incapacidade um dos achados consequentes mais predominantes.

\section{DISCUSSÃO}

Com o aumento da expectativa de vida e diminuição da taxa de fecundidade, o número de idosos vem crescendo significativamente em todo o território brasileiro e isso têm mudado a estrutura da população, deixando de ser primariamente um país de jovens para uma acessão de idosos ${ }^{6}$.

As quedas são eventos bastante comuns durante a velhice, deixando a maioria dos idosos com medo de reincidência. Isso acontece por diversos fatores que vão desde a fragilidade da idade até a estrutura do interior de suas próprias casas ou saídas em passeio. Sendo assim, constituem-se um dos problemas de saúde pública presente nos últimos tempos, havendo progressivamente o aumento de casos, que podem levá-los, desde sequelas a morte ${ }^{7}$.

A queda e as complicações dela decorrentes são as principais causas de morte na terceira idade. De acordo com pesquisas, aproximadamente $30 \%$ das pessoas com mais de 65 caem ao menos uma vez por ano. A consequência mais temida das quedas é a fratura, principalmente a do colo do fêmur, pois ela exige tratamento cirúrgico e um longo período de repouso ${ }^{8}$.

Os fatores de risco intrínsecos às quedas são aqueles de ordem fisiológica ou patológica acompanhando o processo natural de envelhecimento, causando inabilidade ou prejuízo na estrutura corporal e na funcionalidade, impossibilitando a rapidez do comando dos reflexos posturais. Já os extrínsecos são representados pelos fatores ambientais onde o idoso vive, tais como: objetos espalhados pela casa, presença de animais domésticos, falta de acessibilidade, entre outros ${ }^{9}$.

Destacam-se ainda ambientes inseguros ou de risco aqueles que se apresentam com pouca iluminação, tapetes espalhados pelo meio do caminho, pisos escorregadios e encerado, vasos sanitários, sofás e cadeiras muitos baixos, assim como cama demasiadamente baixa ou alta e calçados mal adaptados ${ }^{10}$

Kikucha $^{11}$, ao analisar os fatores associados às quedas em idosos institucionalizados, constatou a prevalência de tontura e desequilíbrio, uso de medicamentos, escorregões, dor, fraqueza muscular, hemiplegia, tropeços, calçado inadequado. Além disso, idosos com histórico de queda estavam mais susceptíveis à recidiva.

Os idosos negam sentir os efeitos do envelhecimento em suas vidas, por isso não atentam a esses fatores e riscos. Neste contexto, a porção majoritária destes resiste ao enfrentamento dos problemas que decorrem da desaceleração natural que acontece nessa fase da vida como, por exemplo, o decréscimo do sistema neuromuscular, a perda de massa muscular, a redução da flexibilidade, da força, da resistência e da mobilidade, além da limitação da capacidade de coordenação e 
Quadro 1: Guia de integração dos artigos.

\begin{tabular}{|c|c|c|c|c|}
\hline Autores/ano & Titulo & Objetivo & Resultados & $\begin{array}{l}\text { Base de } \\
\text { Dados }\end{array}$ \\
\hline $\begin{array}{l}\text { Kikucha et. al., } \\
\text { 2010. }\end{array}$ & $\begin{array}{l}\text { Fatores Associados a Quedas Durante a } \\
\text { Utilização de Escadas por Idosos } \\
\text { Institucionalizados }\end{array}$ & $\begin{array}{c}\text { Analisar os fatores associados às quedas } \\
\text { durante a utilização de escadas pelos } \\
\text { residentes de uma instituição de longa } \\
\text { permanência para idosos }\end{array}$ & $\begin{array}{l}\text { Este estudo demonstrou que as quedas durante a utilização das } \\
\text { escadas pelos idosos institucionalizados podem ser provocadas } \\
\text { principalmente por fatores intrínsecos }\end{array}$ & Scielo \\
\hline $\begin{array}{l}\text { Oliveira et. al., } \\
2011\end{array}$ & $\begin{array}{l}\text { Análise comparativa do risco de quedas } \\
\text { entre pacientes com e sem diabetes } \\
\text { mellitus tipo } 2\end{array}$ & $\begin{array}{l}\text { Comparar a frequência e o risco de } \\
\text { quedas baseado em teste de mobilidade } \\
\text { funcional }\end{array}$ & $\begin{array}{l}\text { Segundo esta pesquisa, não houve diferença estatística entre o } \\
\text { número de quedas para os grupos de idosos estudados. Entretanto, os } \\
\text { não diabéticos obtiveram melhor desempenho quando observadas as } \\
\text { categorias de risco descritas. A redução da acuidade visual e a } \\
\text { dificuldade para levantar foram mais referidas }\end{array}$ & Scielo \\
\hline $\begin{array}{l}\text { Barros et. al., } \\
\quad 2015\end{array}$ & $\begin{array}{l}\text { Reflexões sobre a autonomia do idoso } \\
\text { institucionalizado: uma revisão de } \\
\text { literatura. }\end{array}$ & $\begin{array}{l}\text { Refletir, a partir da literatura, sobre o } \\
\text { cuidado com a autonomia do idoso } \\
\text { institucionalizado. }\end{array}$ & $\begin{array}{l}\text { De acordo com este trabalho é preciso oferecer um cuidado que } \\
\text { priorize a escuta ativa, de forma que, por meio desta prática, os } \\
\text { profissionais tornem-se conhecedores sensíveis dos anseios dos } \\
\text { idosos subsidiando a oportunidade do autêntico encontro de cuidado, } \\
\text { em que tanto o"eu? (cuidador) e o idoso, sujeito de cuidado, } \\
\text { participem ativamente desta permutação }\end{array}$ & Scielo \\
\hline Schneider, 2012 & $\begin{array}{l}\text { Envelhecimento e quedas: a fisioterapia } \\
\text { na promoção e atenção à saúde do idoso }\end{array}$ & $\begin{array}{l}\text { Estudar o processo de envelhecimento e } \\
\text { compreender o acometimento das } \\
\text { funções neuromotoras que interferem na } \\
\text { mobilidade e flexibilidade do idoso com } \\
\text { relação às quedas }\end{array}$ & $\begin{array}{l}\text { Neste estudo percebe-se a relevância do tema abordado, bem como } \\
\text { suas repercussões na qualidade de vida da população idosa, e a } \\
\text { importância da atuação do fisioterapeuta, enfatizado pelo seu papel } \\
\text { enquanto promotor de saúde e equipe de reabilitação. }\end{array}$ & Scielo \\
\hline $\begin{array}{l}\text { Recanelo et. al., } \\
2014\end{array}$ & $\begin{array}{l}\text { Repercussões das quedas na vida dos } \\
\text { idosos e seus familiares }\end{array}$ & $\begin{array}{l}\text { Compreender as repercussões das } \\
\text { quedas na vida dos idosos e seus } \\
\text { familiares a partir de suas perspectivas. }\end{array}$ & $\begin{array}{c}\text { Este estudo revelou que as quedas ocasionam repercussões } \\
\text { semelhantes na vida dos idosos que caem e na vida dos familiares } \\
\text { que vivenciam a queda. }\end{array}$ & Scielo \\
\hline Maciel, 2010 & $\begin{array}{l}\text { Quedas em idosos: um problema de } \\
\text { saúde pública desconhecido pela } \\
\text { comunidade e negligenciado por muitos } \\
\text { profissionais da saúde e por autoridades } \\
\text { sanitárias brasileiras }\end{array}$ & $\begin{array}{l}\text { Chamar a atenção para a intensidade e a } \\
\text { gravidade desse problema. }\end{array}$ & $\begin{array}{c}\text { Este estudo demonstrou que as quedas na idade avançada } \\
\text { representam grave problema de saúde pública, tratado como } \\
\text { circunstância menor por muitos profissionais e gestores da saúde } \\
\text { brasileiros. }\end{array}$ & Scielo \\
\hline $\begin{array}{l}\text { Prado, et. al., } \\
2010\end{array}$ & $\begin{array}{l}\text { A influência dos exercícios resistidos no } \\
\text { equilíbrio, mobilidade funcional e na } \\
\text { qualidade de vida de idosas }\end{array}$ & $\begin{array}{l}\text { Avaliar o equilíbrio, a mobilidade } \\
\text { funcional e a qualidade de vida de } \\
\text { idosas }\end{array}$ & $\begin{array}{l}\text { Este estudo demonstrou o programa de exercícios resistidos } \\
\text { (conhecidos como musculação) utilizado foi eficaz no aumento do } \\
\text { equilíbrio, mobilidade funcional e domínio físico e psicológico da } \\
\text { qualidade de vida das idosas. }\end{array}$ & BVS \\
\hline $\begin{array}{l}\text { Padoin et al., } \\
\quad 2010\end{array}$ & $\begin{array}{l}\text { Análise comparativa entre idosos } \\
\text { praticantes de exercício físico e } \\
\text { sedentários quanto ao risco de quedas }\end{array}$ & $\begin{array}{l}\text { Avaliar o risco de quedas em idosas que } \\
\text { praticam atividades físicas e idosas } \\
\text { sedentárias }\end{array}$ & $\begin{array}{l}\text { Este estudo demonstrou que as idosas sedentárias apresentaram } \\
\text { menor mobilidade funcional, maiores déficits no equilíbrio e } \\
\text { alterações na marcha quando comparadas a idosas que praticavam } \\
\text { exercício físico regularmente. Dessa forma, como resultado a prática } \\
\text { de exercício físico contribuiu para a redução do risco de quedas. }\end{array}$ & BVS \\
\hline $\begin{array}{l}\text { Celich, et al. } \\
\quad 2010\end{array}$ & $\begin{array}{l}\text { Fatores que predispõem às quedas em } \\
\text { idosos }\end{array}$ & $\begin{array}{l}\text { Identificar os fatores que predispõem às } \\
\text { quedas em idosos determinando a } \\
\text { influência dessas intercorrências em } \\
\text { suas vidas }\end{array}$ & $\begin{array}{l}\text { Este estudo demonstrou que são inúmeros os fatores predisponentes } \\
\text { de quedas em idosos, os quais interagem como agentes } \\
\text { determinantes tanto para quedas acidentais quanto para quedas } \\
\text { recorrentes. A prevenção de quedas deve ser visualizada por todos os } \\
\text { profissionais e gestores como mais um desafio na saúde pública, } \\
\text { justamente pelos prejuízos e morbidade relacionados e suas } \\
\text { implicações para aspectos importantes da saúde geral }\end{array}$ & Scielo \\
\hline
\end{tabular}

Fonte: dados da pesquisa, 2016.

do controle do equilíbrio corporal, que podem acarretar quedas frequentes ${ }^{12}$

Fundamentalmente a reformulação de modelo tecnoassistencial sugerida pelo governo para cuidar do idoso baseia-se na mudança do olhar sobre o indivíduo e sua patologia para o cuidado integral das pessoas em seu contexto familiar e comunitário, tendo como base teórica e prática a integralidade da atenção, a promoção da saúde e a vigilância em saúde ${ }^{13}$.

Neste contexto, é essencialmente necessária a promoção à saúde do idoso, a qual deve ser implicada em atividades voltadas a grupos sociais e indivíduos através de políticas públicas abrangentes, incluindo aspectos do ambiente físico, social e político, econômico, cultural e do esforço comunitário, na busca de melhores condições de $\operatorname{vida}^{14-6}$.

Para alcançar uma velhice saudável e diminuir a morbidade é preciso promover a saúde e o bem-estar durante toda a vida do individuo, pois se entende que a saúde é um processo continuado. Para tanto, desde a constituição do SUS em 1988, foram criadas no Brasil políticas voltadas à saúde do idoso que tem como objetivo mantê-los inseridos ao máximo na comunidade, junto à família, da forma mais digna e confortável possível. Entretanto, o que interfere na demanda educativa para essa faixa etária e seu cuidador é a falta de esclarecimento no tocante à prevenção das quedas, que são fatores integrantes e preocupantes no contexto do envelhecimento saudável ${ }^{11}$.

As dificuldades descritas pelos cuidadores também compreendem a espera em filas de supermercados, bancos, ônibus e falta de assentos nos transportes públicos, destacando uma sociedade que não estabeleceu uma importância pelo ser idoso pelo fato do crescimento dessa população ainda ser uma realidade relativamente recente. A incapacidade funcional ocorre à medida que os idosos não conseguem se adaptar às mudanças decorrentes do processo de envelhecimento e ao meio onde vivem ${ }^{15-1}$.

Consequentemente, esses obstáculos provocam um grande impacto sobre a mobilidade, a independência e a qualidade de vida dos idosos e afeta a sua locomoção. Além do ambiente externo, a habitação e o sistema de transporte contribuem para a mobilidade confiante, comportamentos saudáveis, participação social e a determinação, porém o inverso pode provocar o isolamento, a inatividade e a exclusão social ${ }^{12}$.

Observou-se também que após as quedas o idoso tornase mais propício à baixa autoconfiança em realizar suas atividades, seja por medo de novos episódios de queda ou devido a outros fatores físicos, psicológicos ou sociais. Ainda, um comprometimento progressivo da capacidade funcional desse idoso ao longo do tempo, o que pode tornálo mais propenso a quedas recorrentes ${ }^{1}$.

A partir da análise dos fatores que predispõem às quedas, torna-se viável um controle desse problema e, consequentemente, a promoção à população idosa mais funcionalidade, independência, qualidade e aumento da expectativa de vida. No entanto, percebe-se também que a temática é bastante discutida, mas as ações voltadas ao 
combate desse problema ainda são escassas. Com isso, sugerem-se mais discussões voltadas à práxis, isto é, colocar em prática os meios de se combater as quedas, por meio de políticas públicas, por exemplo.

O conhecimento desses fatores de risco e as reflexões acerca da prevenção das quedas também se tornam de suma importância para os profissionais da saúde, uma vez que auxiliam e subsidiam a tomada de decisão diante do paciente idoso.

\section{CONCLUSÃO}

A partir dos estudos selecionados foi demonstrado que fatores intrínsecos e extrínsecos (presença das escadas, problemas de locomoção, diminuição do autocuidado, o papel familiar e, diminuição da qualidade de vida) são relacionados às quedas na população idosa, sendo a incapacidade um dos achados consequentes mais predominantes. As quedas, compreendidas como problemas de saúde pública, necessitam urgentemente da criação de programas com intuito de prevenção, fato este que ainda não é presente na realidade brasileira. Concomitantemente, deve haver capacitação tanto para os profissionais que trabalham nessa esfera como também para os cuidadores, objetivando a melhora no cuidado.

\section{REFERÊNCIAS}

1. Schneider ARS. Envelhecimento e quedas: a fisioterapia na promoção e atenção à saúde do idoso. RBCEH. 2010; 7(2):296-303.

2. Carvalho JA, Garcia RA. O envelhecimento da população brasileira: um enfoque demográfico. Cad Saúde Pública. 2003;19(3):725-33.

3. Menezes R, Bachion MM. Estudo de presença de riscos intrínsecos para queda em idosos institucionalizados. Ciênc saúde coletiva. 2008;13(4):1209-18.

4. Ribeiro AP, Souza ER, Atie S, Souza AC, Schilitz AO. A influência das quedas na qualidade de vida de idosos. Ciênc saúde coletiva. 2008;13(4):1265-73.

5. Alves Junior ED, Paula, FL. A prevenção de quedas sob o aspecto da promoção da saúde. Fit Perf J. 2008;7(2):123-9.

6. Barros MS, Carneiro AD. Reflexões sobre a autonomia do idoso institucionalizado: uma revisão de literatura. Rev Saúde \& Ciência. 2015; 4(3 Supl):51-61.

7. Lopes FL, Tier CG, Lunardi Filho W, Santos SSC. Diagnósticos de enfermagem de idosos residentes em uma instituição de longa permanência (ILP). Cienc Cuid Saúde. 2007; 6(1):59-67.

8. Siqueira FV, Facchini LA, Piccini RX, Tomasi E, Thumé E, Silveira DS, et. al. Prevalência de quedas em idosos e fatores associados. Rev Saúde Pública. 2007;41(5):749-756

9. Recanello CG, Reiners AAO, Azevedo RCS, Alexandre RMS, Cegati L. Repercussões da quedas na vida dos idosos e seus familiares. Rev enferm UFPE on line. 2015; 9(3):7111-7.

10. Maciel A. Quedas em idosos: um problema de saúde pública desconhecido pela comunidade e negligenciado por muitos profissionais da saúde e por autoridades sanitárias brasileiras. Rev Med Minas Gerais 2010; 20(4):554-7.

11. Kikuca EH, Bispo NNC. Fatores Associados a Quedas Durante a Utilização de Escadas por Idosos Institucionalizados. UNOPAR Cient Ciênc Biol Saúde. 2010; 12(2):45-50.
12. Prado RA, Egydio PRM, Teixeira ALC, Izzo P, Langa CJSO. A influência dos exercícios resistidos no equilíbrio, mobilidade funcional e na qualidade de vida de idosas. O mundo da saúde. 2010; 34(2):183-91.

13. Timo-Iara C. O Envelhecimento, Acta Fisiátrica. 2003; 10(3):114-20.

14. Celich KLS, Souza SMS, Zenevicz L, Orso ZA. Fatores que predispõem às quedas em idosos. RBCEH. 2010; 7(3):419-26.

15. Paidon PG, Gonçalves MP, Comaru T, Silva AMV. Análise comparativa entre idosos praticantes de exercício físico e sedentários quanto ao risco de quedas. O mundo da saúde. 2010; 34(2):158-64.

\section{CONFLITO DE INTERESSES}

Os autores declaram não haver conflitos de interesse.

\section{AUTOR PARA CORRESPONDÊNCIA}

\section{Yago Tavares Pinheiro}

yagotavares5@gmail.com

Submetido em 31/10/2016 Aceito em 05/12/2016 